\title{
Measurements on rotating ion cyclotron range of frequencies induced particle fluxes in axisymmetric mirror plasmas
}

\author{
R. Hatakeyama, ${ }^{\text {a) }}$ N. Hershkowitz, R. Majeski, ${ }^{\text {b) }}$ Y. J. Wen, ${ }^{\text {c) }}$ D. B. Brouchous, ${ }^{\text {d) }}$ \\ P. Proberts, ${ }^{\text {() }}$ R. A. Breun, D. Roberts, ${ }^{\text {f) }}$ M. Vukovic, ${ }^{\text {() }}$ and T. Tanaka ${ }^{\text {h) }}$ \\ Department of Nuclear Engineering and Engineering Physics, University of Wisconsin, Madison, \\ Wisconsin 53706
}

(Received 23 January 1997; accepted 2 April 1997)

\begin{abstract}
A comparison of phenomenological features of plasmas is made with a special emphasis on radio-frequency induced transport, which are maintained when a set of two closely spaced dual half-turn antennas in a central cell of the Phaedrus-B axisymmetric tandem mirror [J. J. Browning et al., Phys. Fluids B 1, 1692 (1989)] is phased to excite electromagnetic fields in the ion cyclotron range of frequencies (ICRF) with $m=-1$ (rotating with ions) and $m=+1$ (rotating with electrons) azimuthal modes. Positive and negative electric currents are measured to flow axially to the end walls in the cases of $m=-1$ and $m=+1$ excitations, respectively. These parallel nonambipolar ion and electron fluxes are observed to be accompanied by azimuthal ion flows in the same directions as the antenna-excitation modes $m$. The phenomena are argued in terms of radial particle fluxes due to a nonambipolar transport mechanism [Hojo and Hatori, J. Phys. Soc. Jpn. 60, 2510 (1991); Hatakeyama et al., J. Phys. Soc. Jpn. 60, 2815 (1991), and Phys. Rev. E 52, 6664 (1995)], which are induced when azimuthally traveling ICRF waves are absorbed in the magnetized plasma column.

(C) 1997 American Institute of Physics. [S1070-664X(97)00108-0]
\end{abstract}

\section{INTRODUCTION}

In order to produce, heat and stabilize plasmas by the application of radio-frequency (rf) electromagnetic fields, it is useful to control spatial profiles of the waves excited, and externally determine wave numbers parallel and perpendicular to background magnetic-field lines or preferred directions of wave propagation. For this purpose rotating if systems have often been employed, where the rf field excited preferentially takes the form of a helically traveling wave with poloidal and toroidal phase velocities in a toroidal plasma or with azimuthal and axial phase velocities in a cylindrical plasma. By controlling azimuthal mode numbers $m$ of rotating-rf fields in the ion cyclotron range of frequencies (ICRF), plasma production and heating have efficiently been performed, ${ }^{1}$ and flute instabilities have successfully been stabilized $^{2}$ in magnetized plasma columns.

When such rotating-rf fields are absorbed in the plasma, on the other hand, it is expected that the momentum of this traveling wave is transferred to the plasma particles and a driving force acting along the wave propagation direction appears. The effect of this momentum transfer leads to the generations of steady-state current ${ }^{3}$ and plasma rotation ${ }^{4}$ parallel to the magnetic-field lines, and may induce perpendicu-

\footnotetext{
a) On leave from Department of Electronic Engineering, Tohoku University, Sendai 980-77, Japan. Electronic mail: hatake@ecei.tohoku.ac.jp

${ }^{b}$ Present address: Princeton Plasma Physics Laboratory, Princeton, New Jersey 08543.

${ }^{c}$ On leave from Southwestern Institute of Physics, Leshan, Sichuan, People's Republic of China.

d) Present address: Hutchinson Technology Inc., Madison, Wisconsin 53706.

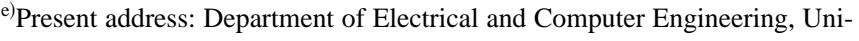
versity of Wisconsin, Madison, Wisconsin 53706.

${ }^{\mathrm{f}}$ Present address: Motorola, Dallas, Texas 75381

${ }^{g}$ Present address: Varian, Palo Alto, California 94304.

${ }^{h}$ Present address: Applied Materials, Santa Clara, California.
}

lar charged-particle fluxes ${ }^{5}$ in the plasma. Concerning the latter point, methods of cross-field flux control by lowfrequency electromagnetic fields were proposed to improve plasma confinement, ${ }^{6}$ kinetic theories on the rf-induced cross-field fluxes in magnetic-mirror and toroidal plasmas were reported, ${ }^{7,8}$ and an orbit theory on the origin of rfinduced particle drifts has been developed. ${ }^{9}$ From an experimental point of view, however, much attention has not been paid to this kind of rf-induced transport. Demirkhanov et al. ${ }^{10}$ observed a change of radial plasma-density profile in a toroidal trap, which depends on the poloidal direction of wave propagation in the lower-frequency Alfvén range $(\omega$ $<\omega_{c i}, \omega / 2 \pi$ : externally applied frequency, $\omega_{c i} / 2 \pi$ : ion cyclotron frequency).

Here our interest is focused on rotating-ICRF-induced particle fluxes in connection with a control of cross-field plasma transport. ${ }^{7,9}$ Being stimulated by our measurements, ${ }^{11}$ experimental trials on this topic have also been made in the Gamma-10 tandem mirror ${ }^{12}$ and small linear ${ }^{13}$ devices, preliminary results of which support our work presented in this paper. In our experiment a set of dual half-turn antennas is used in an axisymmetric tandem mirror to excite rf fields around the ion cyclotron frequency, which azimuthally propagate in the direction of ion cyclotron $(m<0)$ or electron cyclotron $(m>0)$ motion in a plasma. Under such a situation ions are predicted to drift inward and outward in the radial direction in the cases of $m<0$ and $m>0$ excitations, respectively, as clearly explained on the basis of the collisionless orbit theory by Hatakeyama et al. ${ }^{9}$ Then, according to a requirement of the net charge balance,,${ }^{14}$ ion and electron fluxes are expected to be measured at the axial end walls, corresponding to the $m<0$ and $m>0$ excitations, respectively. In the presence of ion collisions a part of the radial drift velocity induced may be transferred to the azimuthal drift velocity. 


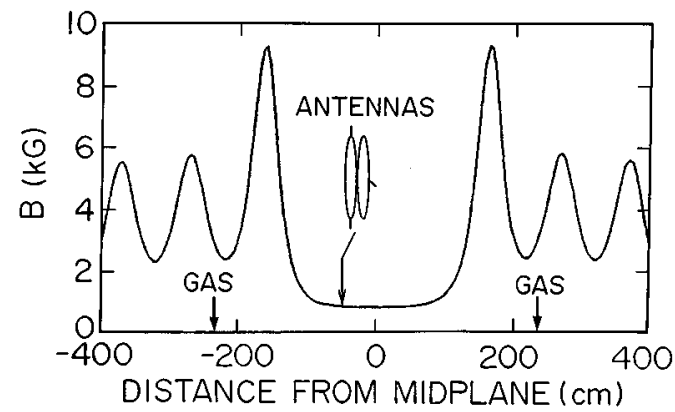

FIG. 1. Schematic of the Phaedrus-B magnetic-field configuration and location of the rotating field phased antenna set.

In Sec. II, the experimental setup and methods are described. Experimental results are presented in Sec. III. Sections IV and V contain discussion and conclusions, respectively.

\section{EXPERIMENTAL SETUP AND METHODS}

Experiments are carried out in the Phaedrus-B tandem mirror consisting of five axisymmetric mirror cells, ${ }^{15}$ as shown in Fig. 1. In the present experiment there is no rf excitation in the plug regions; the device is heated, sustained, and stabilized using only the central cell rf fields. All of the magnets are energized in order to provide mapping of the plasma to the east and west end walls which are electrically grounded. Each end wall consists of five circular concentric segmented electrodes (end rings) whose outer radii are 4.0, $13.0,22.0,30.5$, and $40.5 \mathrm{~cm}$, respectively. The electric current flowing to each ring is measured by passing it through $0.5-\Omega$ resistor. A set of two closely spaced dual half-turn antennas with separation of $7.5 \mathrm{~cm}$ is located at $z$ $=-50 \mathrm{~cm}$ from the central cell midplane (see Fig. 1). The antenna radii are $19 \mathrm{~cm}$ with strap widths of $5 \mathrm{~cm}$ each. There are no Faraday shields, but there are limiters (inner radius $r_{L}: 17 \mathrm{~cm}$, outer radius: $23.5 \mathrm{~cm}$ ) on either side of the antenna set, which are electrically grounded. Two separate amplifier chains capable of providing $200 \mathrm{~kW}$ each are used to excite the antennas with $I_{A}=400 \sim 800 \mathrm{~A}$ (zero-peak value) simultaneously. A single frequency two-phase source is used to drive the chains at a frequency $1.26 \mathrm{MHz}$ which is approximately $\omega_{c i} / 2 \pi$ defined by the magnetic-field strength in the vicinity of the antennas. This variable phase ability allows the antennas to be phased as desired. Thus, the antennas are operated as two closely spaced rotating field antennas, which can be phased to control the azimuthal mode number as previously demonstrated. ${ }^{16}$ By choosing the relative phase between the antenna currents to be $-90^{\circ}$ or $+90^{\circ}$, either the $m=-1$ or the $m=+1$ mode can be preferentially excited with $80 \%$ of the power contained in the excited mode and with the additional power in higher-order modes.

The plasma is created by applying microwaves of 5.5 $\mathrm{GHz}$ and $13 \mathrm{~kW}$, which are resonant near $z= \pm 125 \mathrm{~cm}$ from the central cell midplane, for $3 \mathrm{~ms}$. Hydrogen gas is supplied from gas boxes located at $z= \pm 232 \mathrm{~cm}$ to the vacuum chamber $(\sim 60 \times 2$ Torr $\ell / s)$, in the central region of which the neutral gas pressure is $(0.5-1) \times 10^{-5}$ Torr while the background pressure is below $1.0 \times 10^{-7}$ Torr. After the plasma is initiated, the central cell rf fields are applied, and the plasma duration is up to $20 \mathrm{~ms}$.

Various diagnostics are available. Ion saturation Langmuir probes, double probes, and self-emissive probes are used to determine radial profiles of plasma density $n$, electron temperature $T_{e}$, and plasma potential $\phi$, respectively. A $0.15 \mathrm{~cm}$ diam paddle probe, which is buried in the $0.16 \mathrm{~cm}$ width ceramic support to yield a function similar to a directional Mach probe ${ }^{17}$ and a Gundestrup probe, ${ }^{18}$ is used to estimate an azimuthal anisotropy of plasma flux by measuring a difference of ion saturation currents when its collector faces in the directions of ion cyclotron and electron cyclotron motions. Axial profiles of plasma line density and pressure are obtained using diamagnetic loops and microwave interferometers. The ion temperature $T_{i \perp}$ perpendicular to the magnetic-field lines in the central cell is determined by a time-of-flight analyzer of charge exchange neutral particles. The source (ionization) current is determined from the absolutely calibrated $\mathrm{H}_{\alpha}$ emission. A movable gridded endloss analyzer with $4 \mathrm{~cm}$ spaced 17 holes $(0.6 \mathrm{~cm}$ in diameter $)$ is set just in front of each of the end rings at $z= \pm 535 \mathrm{~cm}$. Since the analyzer can be turned on a fixed point at its edge, the frontal grid of which is electrically grounded, this Faraday-cup array measures cross-sectional profiles of charged particle fluxes and temperatures parallel to the magnetic-field lines $\left(T_{e \|}, T_{i \|}\right)$. The plasma parameters obtained on the axis of the central cell are $n \simeq 3$ $\times 10^{12} \mathrm{~cm}^{-3}, \quad T_{e} \simeq T_{i}=60-90 \mathrm{eV}$ and radius $\simeq 16 \mathrm{~cm}$ ( wall radius $=70 \mathrm{~cm}$ ).

\section{EXPERIMENTAL RESULTS}

According to previous investigations on the stability of the Phaedrus-B tandem mirror, the plasma has been stabilized against magnetohydrodynamics (MHD) interchange activity by the ponderomotive force of the ICRF fields under the condition of $\omega / \omega_{c i}<1$ for $m=-1$ and $\omega / \omega_{c i}>1$ for $m=+1$ while it has been unstable under the condition of $\omega / \omega_{c i}<1$ for $m=+1 .^{16,19}$ In the present experiment, however, hydrogen gas fuelling is provided by the gas boxes located beyond the central cell in order to obtain a stable plasma even under the condition of $\omega / \omega_{c i}<1$ for $m=+1$ (gas-blanket line tying), ${ }^{20}$ the amount of which is larger than in the usual Phaedrus-B experiments by approximately an order of magnitude.

Temporal evolutions of line density near the central-cell midplane $(z=-12.5 \mathrm{~cm})$ and total plasma flux flowing into the end walls (rings) are measured when the rf antenna is phased to excite the rotating ICRF fields with $m=-1$ and $m=+1$, as given in Fig. 2. Significant difference in linedensity behavior is not found for variations of $m$ and $\omega / \omega_{c i}$. However, the net plasma flux along the magneticfield lines at the end walls shows ion (positive) and electron (negative) currents in the cases of $m=-1$ and $m=+1$ excitations, respectively, whenever the rf azimuthal mode is changed with $\omega / \omega_{c i}$ kept constant $(=0.97)$ and for different values of $\omega / \omega_{c i}$ [just above (1.09) or below unity (0.90)]. 


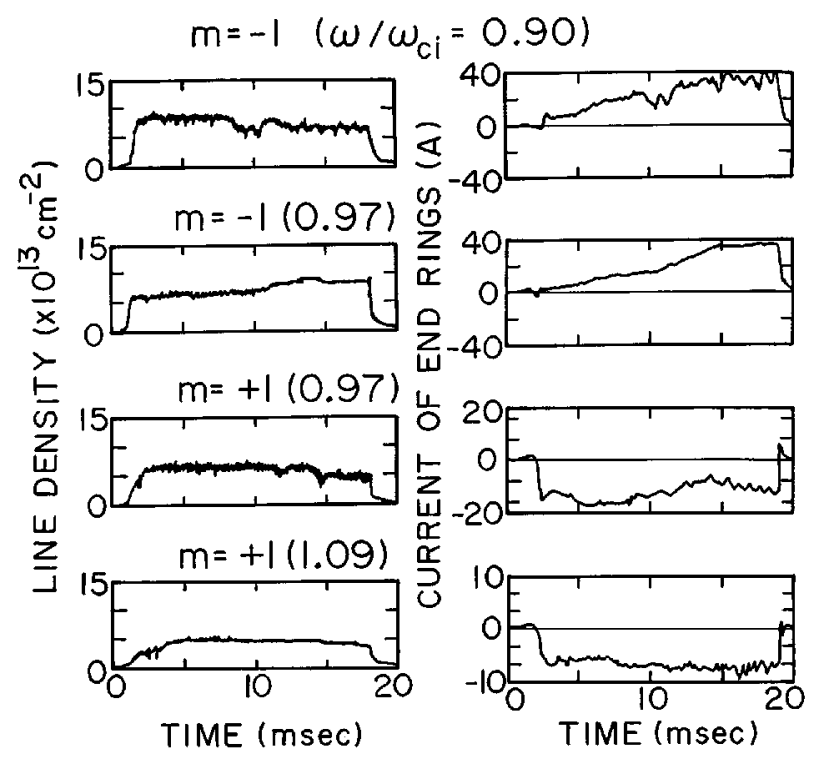

FIG. 2. Temporal variations of line density at $z=-12.5 \mathrm{~cm}$ and total current of the end rings for the cases where the rotating-ICRF excitations are performed with typical values of $\omega / \omega_{c i}$.

Here $\omega / \omega_{c i}$ is varied by changing the magnetic-field strength with the mirror ratios kept almost constant. The radial profile of endloss current is obtained by plotting the electric current of each of the segmented end rings at $t=10-15 \mathrm{~ms}$, as

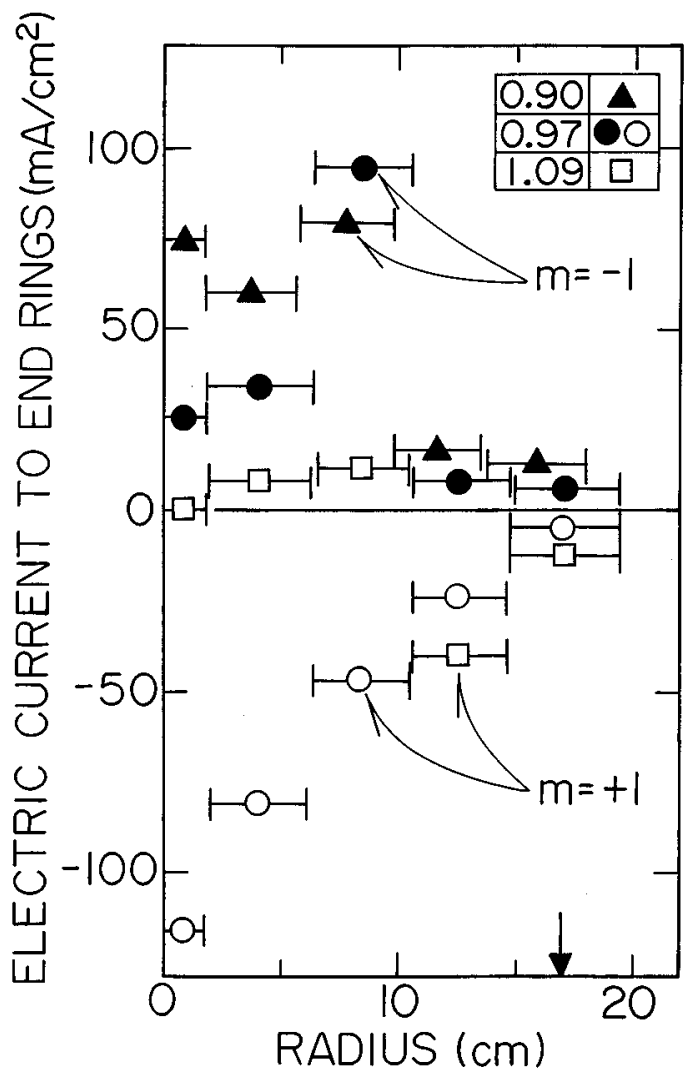

FIG. 3. Radial profiles of current density at the end walls with $\omega / \omega_{c i}$ $=0.90,0.97$, and 1.09. Closed and open marks denote values in the cases of $m=-1$ and +1 excitations, respectively.

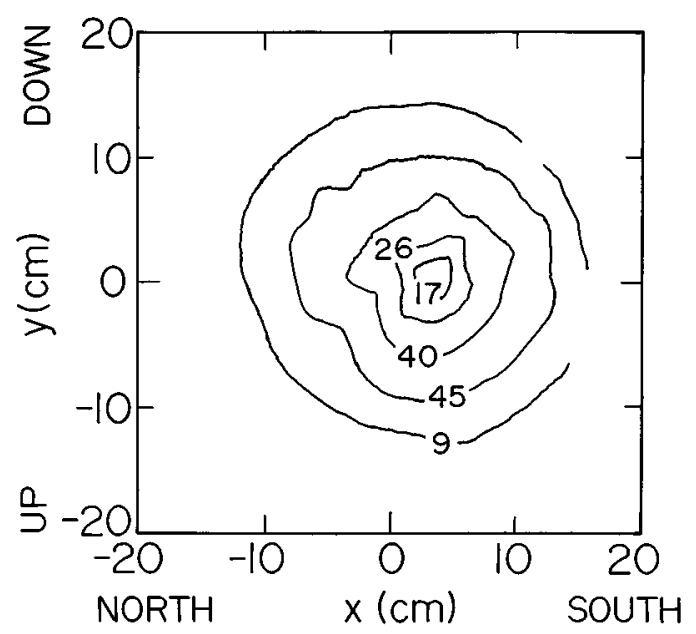

FIG. 4. A typical example of contour plot of charged-particle endloss mapped back to the central cell. Ion current (in $\mathrm{mA} / \mathrm{cm}^{2}$ ) by the west endloss analyzer in the case of $m=-1$ excitation. $\omega / \omega_{c i}=0.97$ and $t=14.9 \mathrm{~ms}$.

shown in Fig. 3, where the radial distance and the current per unit area are evaluated in central-cell coordinates and an arrow indicates the limiter position. The ion-loss current for the $m=-1$ mode is almost of the same magnitude as the electron-loss current for the $m=+1$ mode. In most cases the currents in the core region are larger than in the edge region. The generation of these ion- and electron-loss currents is consistent with the measured result that the potentials of the end rings in the case of $m=-1$ become higher than those in the case of $m=+1$ when they are switched from electrically grounded to floating.

Since operations of the gridded analyzers just in front of the west and east end walls enable us to make cross-sectional contour plots of ion and electron current densities as typically exemplified in Fig. 4, we can decompose the total endloss current into the ion and electron currents to estimate ambipolar $\left(\Gamma_{\|}^{A}\right)$ and nonambipolar $\left(\Gamma_{\|}^{N A}\right)$ current fluxes parallel to the magnetic-field lines. The typical result is $\Gamma_{\|}^{A}$ $\simeq 65 \mathrm{~A}, \Gamma_{i \|}^{N A} \simeq 35 \mathrm{~A}$, and $\Gamma_{\|}^{A} \simeq 50 \mathrm{~A}, \Gamma_{e \|}^{N A} \simeq 30 \mathrm{~A}$ in the cases of $m=-1$ and +1 excitations, respectively, while the plasma source current determined from radial and axial profiles of $\mathrm{H}_{\alpha}$-emission intensity is $\Gamma_{\text {source }} \simeq 500 \mathrm{~A}$ for the both modes. The difference between $\Gamma_{\text {source }}$ and $\Gamma_{\|}$is ascribed to the current flux perpendicular to the magnetic-field lines, which consists of ambipolar $\left(\Gamma_{\perp}^{A}\right)$ and nonambipolar $\left(\Gamma_{\perp}^{N A}\right)$ components. Under our experimental condition, where the large amount of hydrogen gas fuelling is provided, the radial losses in the long regions between the central-cell midplane and the end walls dominate the axial losses at the end walls. It is to be noted that almost invariably negative currents were measured at the end walls in discharges of the tandem mirror TMX-U ${ }^{14}$ and Gamma-10, ${ }^{21}$ where asymmetric applications of electron-cyclotron and neutral beam heating powers are considered one of the candidates for nonambipolar transports observed. $^{22}$

When the nonambipolar perpendicular flux $\Gamma_{\perp}^{N A}$ is considered, it is necessary to make an experimental comparison between azimuthal asymmetries in the plasmas ${ }^{23}$ maintained by the rf excitations with $m=-1$ and +1 modes. For that 

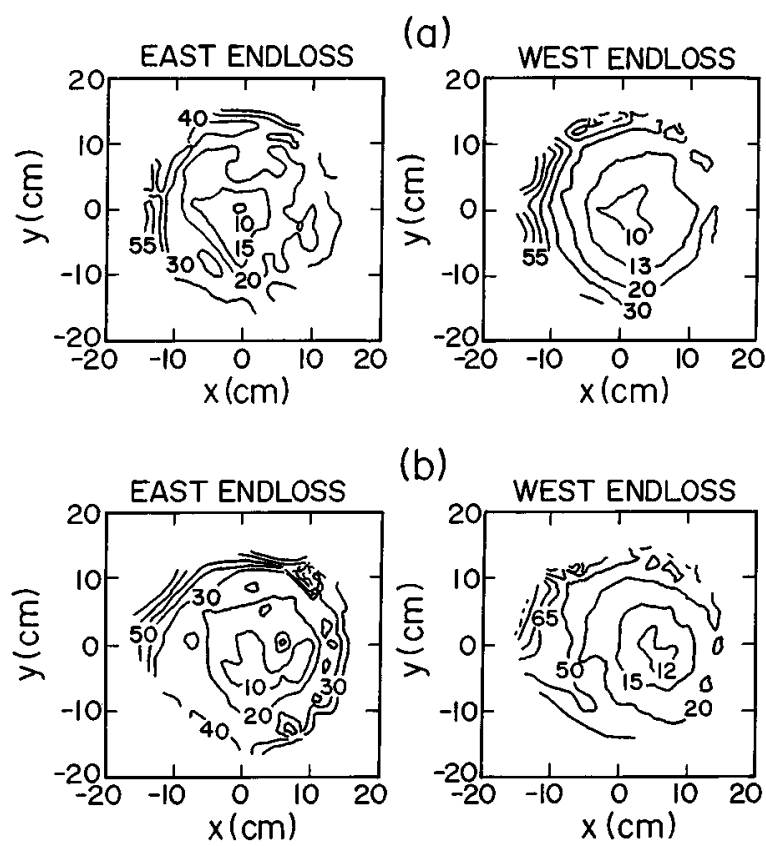

(b)

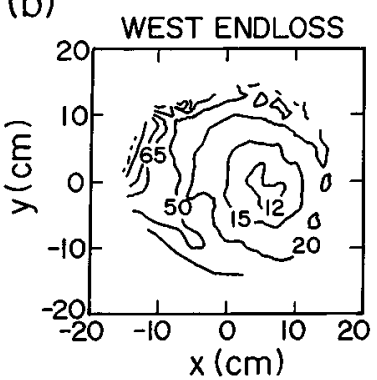

FIG. 5. Contour plots of endloss electron temperature (in $\mathrm{eV}$ ) in the cases of (a) $m=-1$ and (b) +1 excitations (mapped back to the central cell). $\omega / \omega_{c i}=0.97$ and $t=10 \mathrm{~ms}$.

purpose measurements of two-dimensional contour plots of the endloss electron temperature parallel to the magneticfield lines are performed as shown in Fig. $5\left(\omega / \omega_{c i}\right.$ $=0.97$ ), where the contours at $t=10 \mathrm{~ms}$ are mapped back to the central cell and an asymmetry at the corner of the $x-y$ plane is due to the feeder insertion of the rf antenna. Although results of the east endloss analyzers are more asymmetric than those of the west endloss analyzers, overall $T_{e \|}$-contours denote a similar asymmetry for the both excitations with $m=-1$ and +1 . To make this point clear radial profiles of endloss $T_{e \|}$ in the cases of $m=-1$ and +1 excitations are presented in Fig. 6, where the results for two azimuthal angles $\left(45^{\circ}, 135^{\circ}\right)$ are given. We cannot find an appreciable difference of $T_{e \|}$ profile between the both cases, and increases in $T_{e \|}$ are measured at the outer radii. When we sum up and plot the number of loss electrons over the whole cross sections and during the plasma sustainment ( $\sim 20 \mathrm{~ms}$ ) as a function of $T_{e \|}$, the $T_{e \|}$ yielding the largest number of the electrons in the case of $m=-1$ is almost the same $(\sim 15 \mathrm{eV})$ as that in the case of $m=+1$. The tail $T_{e \|}$ of this histogram in the latter case is larger than in the former case. On the other hand the endloss ion temperatures parallel to the magnetic-field lines are measured to be higher than the electron temperatures and to increase at the outer edge similarly to the $T_{e \|}$ profiles. According to the ion temperature histogram in Fig. $7 T_{i \|}$ yielding the peak values are around $50 \mathrm{eV}$ for the both excitation modes, and the higher tail is more enhanced in the case of $m=+1$ similarly to the $T_{e \|}$ histogram. The peak value in the case of $m=-1$ is larger by approximately nine times than that in the case of $m=+1$. Thus, the polarity difference of endloss currents between the $m=-1$ and +1 excitations are unlikely to be connected with the $T_{e \|^{-}}$and $T_{i \|}$-profile properties.

Figure 8 gives time-averaged $(13 \leqslant t \leqslant 16 \mathrm{~ms})$ radial pro-
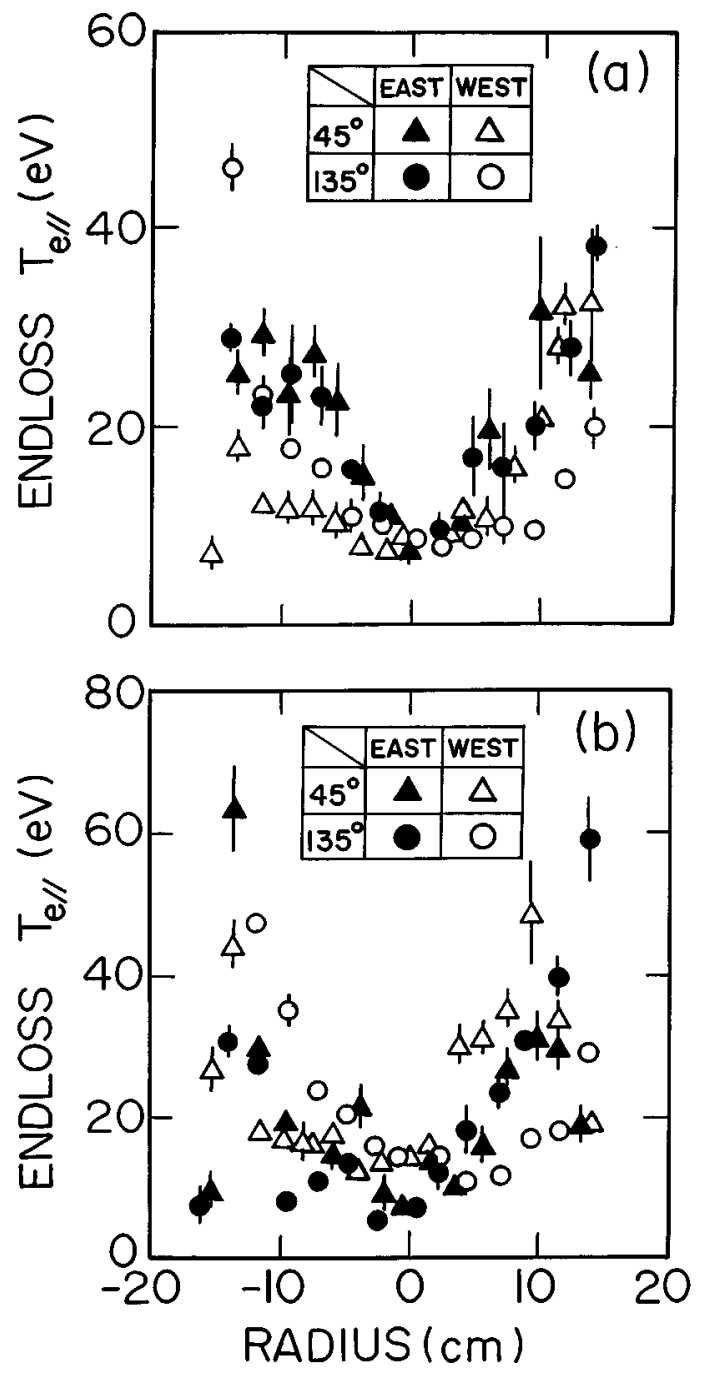

FIG. 6. Radial profiles of endloss electron temperature for typical azimuthal angles at the both ends (east and west). (a) $m=-1$ and (b) $m=+1$. $\omega / \omega_{c i}=0.97$ and $t=10 \mathrm{~ms}$.

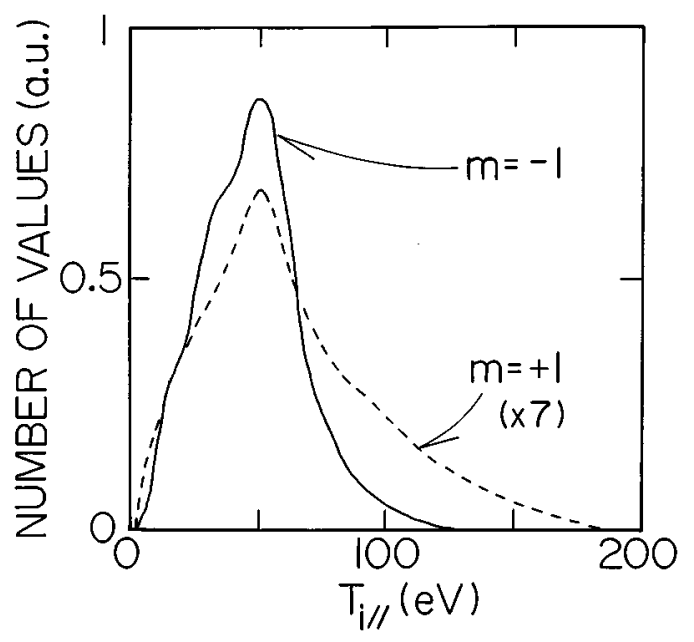

FIG. 7. Histograms of endloss ion temperature for the both excitation modes. $\omega / \omega_{c i}=0.97$. 

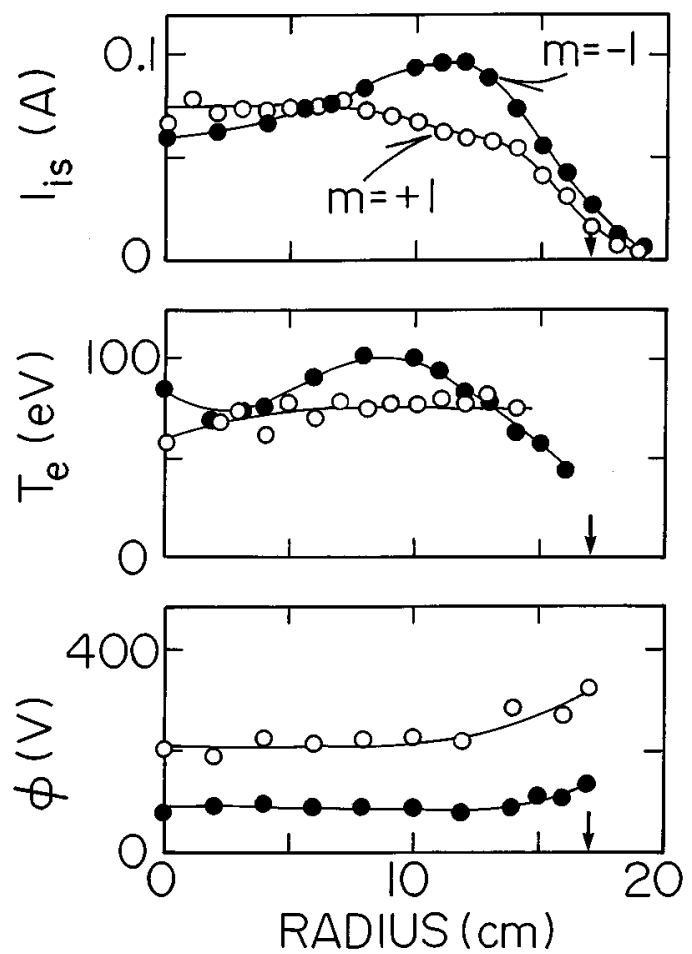

FIG. 8. Radial profiles of ion saturation current, electron temperature, and plasma potential in the central cell $(z=-25 \mathrm{~cm})$. Closed and open circles denote values in the cases of $m=-1$ and +1 excitations. $\omega / \omega_{c i}=0.97$.

files of ion saturation current $I_{i s}$ (proportional to $n$ ), $T_{e}$, and $\phi$ around the midplane in the central cell $(z=-25 \mathrm{~cm})$. The $n$ profile in the case of $m=-1$ is measured to be weakly hollow with an inward diffusion coefficient at $r \lesssim 11 \mathrm{~cm}$ and outward diffusion coefficient at $r>11 \mathrm{~cm}$, while that in the case of $m=+1$ to be more diffusive in the outward direction. Almost the same $T_{e}$ profiles for the both rf-excitation modes are measured by the double probe which preferentially collects higher-tail electrons. The plasma potential is measured to be almost constant in the core region $(r$ $<15 \mathrm{~cm}$ ) and increases slightly in the edge region $(r$ $\gtrsim 15 \mathrm{~cm}$ ) for the both modes. Here $\phi$ in the case of $m$ $=-1$ is lower than in the case of $m=+1$. This result is not in contradiction to the observations in Figs. 2 and 3, where more ions than electrons and more electrons than ions are lost to the end walls in the former and latter cases, respectively. The charge-exchange analyzer in the central cell $(z$ $=75 \mathrm{~cm}$ ) measures perpendicular ion energy distribution functions with bulk and higher-tail components. Figure 9 indicates time variations of bulk and tail $T_{i \perp}$ (upper graph) and of tail density fractions (bottom graph). The tail $T_{i \perp}$ in the case of $m=-1$ is a little bit larger than that in the case of $m=+1$, although the bulk $T_{i \perp}$ are almost same in the both excitation modes. The higher-tail fraction is more pronounced in the case of $m=-1$.

Figure 10 shows time variations of the ion saturation currents measured by the paddle probe in the central cell $(z$ $=25 \mathrm{~cm}, r=9 \mathrm{~cm})$, the collector of which faces in the directions of ion cyclotron $\left(I_{\theta-}\right)$ and electron cyclotron $\left(I_{\theta+}\right)$ motions. It is clearly measured that $I_{\theta-}$ is larger than $I_{\theta+}$ in the case of $m=-1$ but $I_{\theta+}$ is larger than $I_{\theta-}$ in the case of
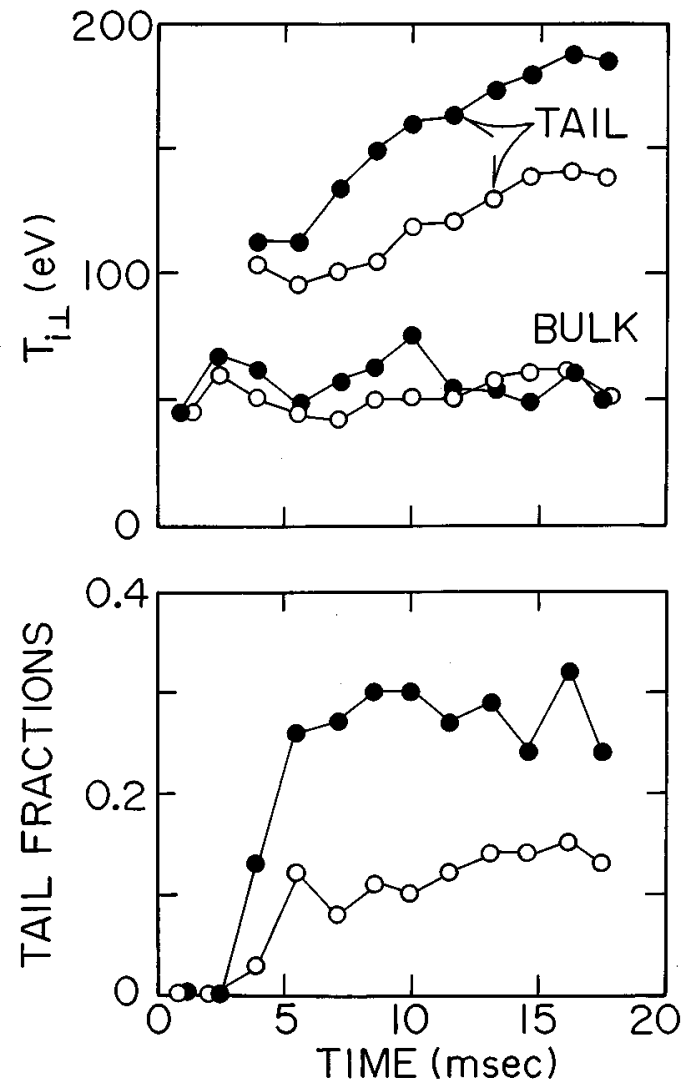

FIG. 9. Time variations of bulk and tail ion temperatures perpendicular to the magnetic field, and of tail density fractions in the central cell $(z$ $=75 \mathrm{~cm}$ ) in the cases of $m=-1$ (closed circles) and +1 (open circles) excitations. $\omega / \omega_{c i}=0.97$.

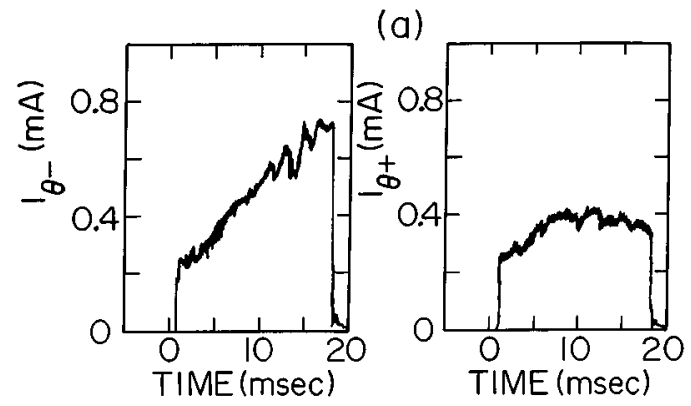

(b)

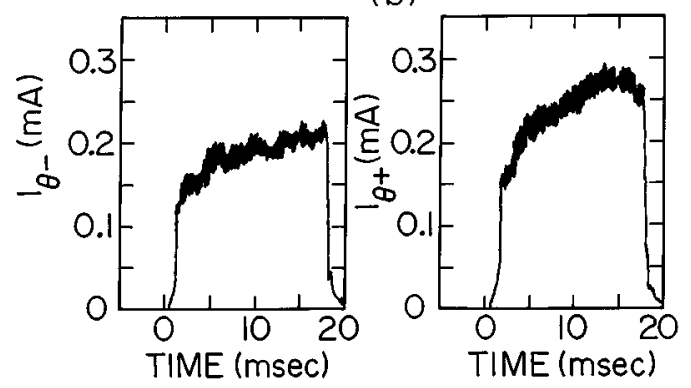

FIG. 10. Time variations of ion saturation currents picked up by the paddle probe faced in the directions of ion $\left(I_{\theta-}\right)$ and electron $\left(I_{\theta_{+}}\right)$cyclotron motions. (a) $m=-1\left(\omega / \omega_{c i}=0.97\right)$ and (b) $m=+1\left(\omega / \omega_{c i}=1.09\right), \quad z$ $=25 \mathrm{~cm}$ and $r=9 \mathrm{~cm}$. 


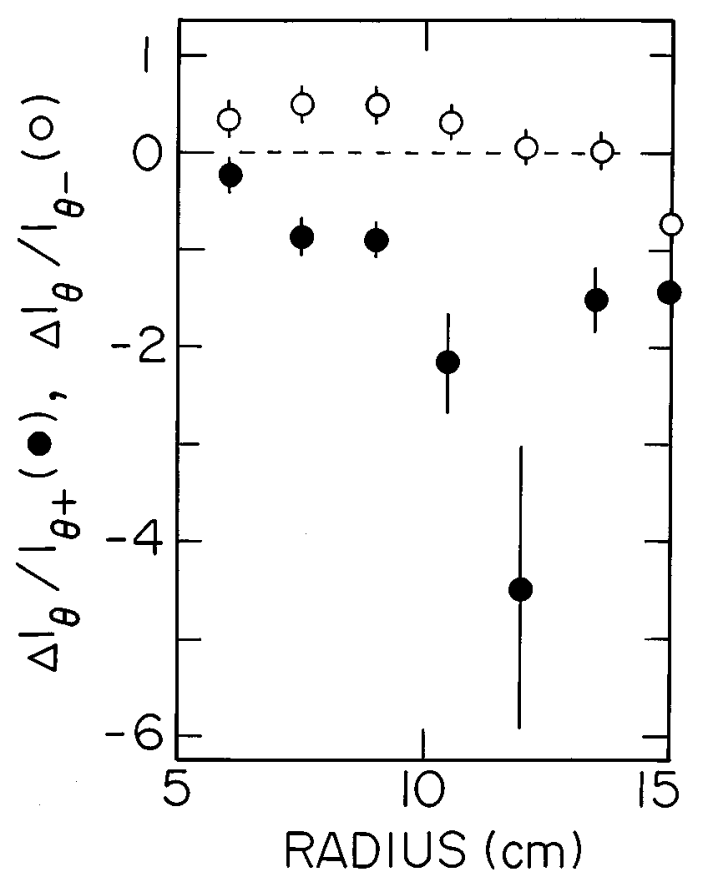

FIG. 11. Radial profiles of normalized difference of paddle-probe ionsaturation currents $\left(\Delta I_{\theta}=I_{\theta+}-I_{\theta^{-}}\right)$in the cases of $m=-1$ (closed circles) and +1 (open circles) excitations. $z=25 \mathrm{~cm}$ and $t=13-16 \mathrm{~ms}$.

$m=+1$. This result indicates that an azimuthal plasma flow is induced in the same direction as the rf excitation mode $m$. Radial profiles of the difference between $I_{\theta+}$ and $I_{\theta-}$ $(13 \leqslant t \leqslant 16 \mathrm{~ms})$ are given in Fig. 11 , where the normalized values of $\Delta I_{\theta} / I_{\theta+}$ for the $m=-1$ excitation and $\Delta I_{\theta} / I_{\theta-}$ for the $m=+1$ excitation are plotted in order to estimate the azimuthal flow speed discussed later $\left(\Delta I_{\theta}=I_{\theta+}-I_{\theta^{-}}\right)$. For the $m=-1$ excitation the ion flow in the direction of ion cyclotron motion is observed in the whole region and has a maximum value around $r=12 \mathrm{~cm}$, although the ion diamagnetic drift velocity is in the opposite direction to ion cyclotron motion at $r \leqq 12 \mathrm{~cm}$. For the $m=+1$ excitation the ion flow in the direction of electron cyclotron motion is observed at $r<15 \mathrm{~cm}$, although the ion diamagnetic drift velocity is in the opposite direction to electron cyclotron motion. The ion flow flux for the $m=+1$ excitation is smaller than that for the $m=-1$ excitation. In the edge inhomogeneous region $(r \gtrsim 15 \mathrm{~cm})$ comparable ion flows in the direction of the ion diamagnetic drift velocity are measured for the both excitation modes. Since the definite difference of the ion flows between the $m=-1$ and +1 excitations is observed in the flat potential-profile region in Fig. 8, where $\mathbf{E}_{r} \times \mathbf{B}$ rotation effects can be neglected, it is clearly attributed to an intrinsic mechanism associated with azimuthally rotating-ICRF properties.

\section{DISCUSSION}

Since the net electron and ion losses integrated over all surfaces must balance under our experimental situation, the nonambipolar endloss fluxes $\Gamma_{\|}^{N A}$ observed can directly be connected with nonambipolar fluxes $\Gamma_{\perp}^{N A}$ perpendicular to the magnetic-field lines. Then radially outward diffusion of
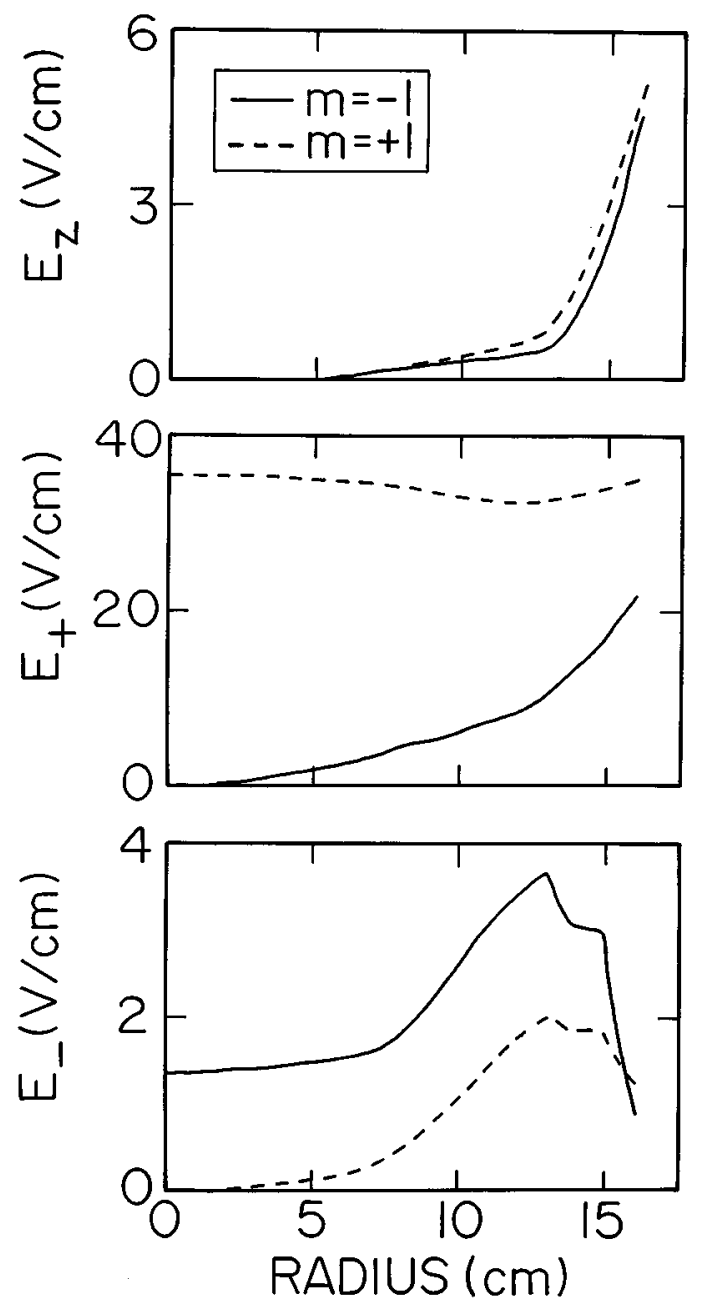

FIG. 12. Results of numerical calculations from the ICRF code ANTENA. Radial profiles of parallel $\left(E_{z}\right)$, right- $\left(E_{+}\right)$and left-hand $\left(E_{-}\right)$polarized components of rf electric field. Solid and dotted curves denote the cases of $m=-1$ and +1 excitations, respectively. $n=3 \times 10^{12} \mathrm{~cm}^{-3}, T_{i}=80 \mathrm{eV}$, $T_{e}=60 \mathrm{eV}, r_{L}=17 \mathrm{~cm}, \omega / \omega_{c i}=1$, and $I_{A}=1000 \mathrm{~A} /$ antenna.

electrons and ions might simply be considered to occur in our plasmas in the cases of $m=-1$ and $m=+1$ excitations, respectively. In order to check effects of rf-field profiles on the observed phenomena numerical calculations from the ICRF code ANTENA ${ }^{24}$ are performed using experimentally determined profiles of $T_{e}, T_{i}$ and $n$. Figure 12 presents calculated profiles of the $\mathrm{rf}$ electric field at $z=0 \mathrm{~cm}$ with $\omega / \omega_{c i}=1.0(B=860 \mathrm{G})$, where $E_{z}, E_{+}$, and $E_{-}$denote the field component parallel to the magnetic field, right-(rotating with electrons) and left-(rotating with ions) hand polarized components, respectively. Since there are no appreciable differences of the $E_{z}$ profile between the both excitation modes, the polarity change of the field-aligned nonambipolar currents observed in Figs. 2 and 3 cannot be explained by the $E_{z}$ effect, which is rather responsible for the appreciable edge heating of the endloss $T_{e \|}$ and $T_{i \|}$ found in Fig. 6. In comparison with the central-cell $T_{e}$ profiles in Fig. 8 it is demonstrated that an enhanced edge heating of $T_{e \perp}$ leading to an outward diffusion of electrons does not occur even in the presence of the radially increasing $E_{+}$for the $m=-1$ 
excitation. Although an enhanced edge heating of $T_{i \perp}$ leading to an outward diffusion of ions is expected to occur due to the radially localized $E_{-}$for the $m=+1$ excitation, this effect may comparatively be negligible because the highertail density and temperature in the case of $m=+1$ are observed to be lower than in the case of $m=-1$, as seen in Fig. 9. Thus, the properties of ion and electron temperatures measured and rf electric fields calculated cannot be related to differences of particle diffusion in the radial direction and resultant axial endloss between the $m=-1$ and +1 excitations.

From a viewpoint of generation mechanism of $\Gamma_{\perp}^{N A}$ let us consider momentum transfer from the azimuthally traveling wave driven by the rotating ICRF antenna. In general, the absorption of $\mathrm{rf}$ fields excited by an external source in a dissipative medium leads to the generation of momentum flow into the constituent particles and thus to the appearance of a force acting along the wave propagation direction. The dissipative force is given by

$$
\mathbf{F}=\sum_{\mathbf{k}} \frac{\mathbf{k}}{\omega(\mathbf{k})} Q(\mathbf{k}),
$$

where $Q(\mathbf{k})$ is the absorbed wave energy in a unit volume of the medium per unit time and the summation is carried out over all the waves. ${ }^{5}$ A clear-cut and useful explanation for the generation mechanism of this force is given under the condition of cyclotron resonances in Ref. 9. In the present case $Q$ is considered mainly due to ion cyclotron damping of the ICRF wave, $\mathbf{k}$ is in the azimuthal direction and thus $\mathbf{F}$ is the azimuthal force acting on ions. When the rf antenna is phased so as to excite the mode with $m=+1, \mathbf{F}_{\theta}$ in the same direction as the electron cyclotron motion may give rise to the radially outward drift of ions in the axial magnetic field $\left(\mathbf{v}_{r}=\mathbf{F}_{\theta} \times \mathbf{B} / e B^{2}\right)$. This $\Gamma_{i \perp}^{N A}$ induced is considered to be canceled by the axial electron flux $\Gamma_{e \|}^{N A}$ observed. When the mode with $m=-1$ is excited, $\mathbf{F}_{\theta}$ in the same direction as the ion cyclotron motion may give rise to radially inward drift of ions in the axial magnetic field. As a result of this $\Gamma_{i \perp}^{N A}$ induced ions coming around the radial center is considered to flow along the magnetic field, yielding the endloss flux $\Gamma_{i \|}^{N A}$ observed. This endloss $\Gamma_{i \|}^{N A}$ must be canceled by a perpendicular electron loss $\Gamma_{e \perp}^{N A}$. Since the difference between the plasma and radial-wall (grounded) potentials is so small $\left(\leq T_{e} / e\right)$ in the case of $m=-1$, as shown in Fig. 8, a part of the electrons may come over the potential barrier, flowing into the radial wall.

In the presence of collisions between ions and the other particles a part of the ion momentum transferred from the azimuthally rotating rf fields described above is expected to be scattered into the radial direction. In order to obtain simple expressions for the momentum scattering we solve the steady-state fluid equation of ion motion in an axisymmetric system of the cylindrical coordinates $(r, \theta, z)$. When effects of radial pressure gradient and ion convective motion are negligible small, the ion flow speeds in the radial and azimuthal directions are given by $\left(\nu_{i} / \omega_{c i}\right) v_{r} \simeq v_{\theta}$ and

$$
v_{\theta} \simeq \frac{F_{\theta}}{e B} \frac{\nu_{i} / \omega_{c i}}{1+\left(\nu_{i} / \omega_{c i}\right)^{2}} .
$$

Here $\nu_{i}$ is the ion collision frequency dominated by the ionneutral collisions $\left(\nu_{i} / \omega_{c i}<0.1\right)$ including elastic collisions $(<150 \mathrm{kHz})$, ionization $(<40 \mathrm{kHz})$ and charge-exchange collisions $(<30 \mathrm{kHz})$ in our case. ${ }^{25}$ Thus, the azimuthal ion flux is produced by the radial flux loss due to the ion collisions, the direction of which is the same as $\mathbf{F}_{\theta}$, i.e., the direction of the antenna-excitation mode $m$ in our experiment. This consideration is consistent with the measured anisotropy of azimuthal ion-saturation currents of the paddle probe in Figs. 10 and 11. When ions are assumed to form a shifted-Maxwellian velocity distribution function with a flow speed $v_{\theta}$ in the azimuthal direction, the differences of the probe saturation currents $\left(I_{\theta+}-I_{\theta-}\right)$ are related to $v_{\theta}(<0$ for $m=-1 ;>0$ for $m=+1)$ by

$$
\frac{\Delta I_{\theta}}{I_{\theta \pm}}=\mp \frac{2 \sqrt{\pi} V_{\theta}}{\sqrt{\pi} V_{\theta}\left[-1+\operatorname{erf}\left(\mp V_{\theta}\right)\right] \mp \exp \left(-V_{\theta}^{2}\right)},
$$

where the upper and lower signs refer to the $m=-1$ and +1 excitations, respectively, $V_{\theta}=v_{\theta} / \sqrt{2 T_{i} / M}, M$ is ion mass, and $\operatorname{erf} x=(2 / \sqrt{\pi}) \int_{0}^{x} \exp \left(-t^{2}\right) d t$. Combining Eq. (3) with Fig. 11 gives $-V_{\theta}<0.5$ and $V_{\theta} \lesssim 0.1$ in the cases of $m=-1$ and +1 excitations, respectively. These values are several times as large as those predicted from Eq. (2), when we assume that the radial flux induced around the central-cell midplane is of the order of the plasma source flux $\left(\left|F_{\theta}\right| / e B \sim \sqrt{2 T_{i} / M}\right)$ because we have no exact theory at present, which determines the absolute value of the momentum transfer from the rotating-ICRF fields to the ions. However, this difference may be compensated for by a moreenhanced momentum scattering into the radial direction when we take effective ion collisions due to low-frequency $\left(<\omega_{c i} / 2 \pi\right)$ fluctuations in the plasmas into account, which are known to cause ambipolar transport. ${ }^{26}$

Finally it is to be noted that the dissipative force given by Eq. (1) may also be due to classical particle collisions even in the absence of kinetic interactions such as Landau and cyclotron dampings. Thus, we cannot completely neglect a possibility that an electron flux in the radial direction is generated due to electron-neutral collisions in the presence of ICRF waves. ${ }^{27}$ In order to quantitatively clarify this effect on the phenomenon observed in our experiment, however, much theoretical work has to be developed, which is beyond the scope of this paper.

\section{CONCLUSIONS}

Plasma features resulting from excitations of ICRF fields have been compared to investigate rf-induced particle fluxes, which azimuthally rotate with ions $(m<0)$ and electrons $(m>0)$ in axisymmetric magnetic-mirror fields. Nonambipolar ion and electron currents are measured at the axial end walls for the excitations with the modes $m=-1$ and +1 , respectively, which require the generation of radial fluxes under the condition of the total flux balance. Momentum transfers from azimuthally traveling ICRF waves to ions are considered to induce radially inward and outward ion drifts in the cases of $m=-1$ and +1 excitations, respectively. On the other hand azimuthal ion fluxes with the same directions as the ICRF excitation modes are also observed, which are 
considered to originate from collision scatterings of the ion momentum transferred from the traveling waves. Our results suggest that ICRF waves could be useful for the control of cross-field fluxes in open-ended, divertor, and toroidal plasmas.

\section{ACKNOWLEDGMENTS}

One of the authors (R.H.) would like to thank Professor N. Hershkkowitz and his collaborators for their kind hospitality and helps during his stay in Madison. He expresses his thanks to Professors N. Sato and M. Inutake for their continuous encouragement and support. He is also grateful for discussions with T. Hatori, H. Hojo, and T. Watanabe.

This work was carried out under the US-Japan Fusion Cooperation Program (QL70).

${ }^{1}$ Y. Yasaka and R. Itatani, Phys. Rev. Lett. 44, 1763 (1980); T. Watari, K. Adati, T. Aoki, S. Hidekuma, K. Hattori, S. Hiroe, M. Ichimura, T. Kawamoto, R. Kumazawa, Y. Okubo, S. Okamura, T. Sato, C. M. Singh, and M. Sugawara, Nucl. Fusion 22, 1359 (1982); S. Okamura, K. Adati, T. Aoki, D. R. Baker, H. Fujita, H. R. Garner, K. Hattori, S. Hidekuma, T. Kawamoto, R. Kumazawa, Y. Okubo, and T. Sato, ibid. 26, 1491 (1986). ${ }^{2}$ J. R. Ferron, N. Hershkowitz, R. A. Breun, S. N. Golvato, and R. Goulding, Phys. Rev. Lett. 51, 1955 (1983); Y. Yasaka and R. Itatani, ibid. 56, 2811 (1986).

${ }^{3}$ R. Klima, Plasma Phys. 15, 1031 (1973); T. Kondoh and the JT-60 Team, Phys. Plasmas 1, 1489 (1994).

${ }^{4}$ S. Wukitch, C. Litwin, M. Harper, R. Parker, and N. Hershkowitz, Phys. Rev. Lett. 77, 294 (1996).

${ }^{5}$ A. Bers, in Plasma Physics, edited by C. DeWitt and J. Peyraud (Gordon and Breach, New York, 1975), p. 126; V. V. Nemov, Fiz. Plazmy 4, 1280 (1978) [Sov. J. Plasma Phys. 4, 714 (1978)].

${ }^{6}$ V. V. Dolgopolov, Fiz. Plazmy 5, 1203 (1979) [Sov. J. Plasma Phys. 5, 672 (1979)]; S. Inoue and K. Itoh, in Plasma Physics and Controlled Nuclear Fusion Research, 1980 (International Atomic Energy Agency, Vienna, 1981), Vol. 2, p. 649; V. P. Minenko and V. P. Sidorov, Fiz. Plazmy 11, 147 (1985) [Sov. J. Plasma Phys. 11, 85 (1985)].

${ }^{7}$ H. Hojo and T. Hatori, J. Phys. Soc. Jpn. 60, 2510 (1991).

${ }^{8}$ M. Rosenberg, N. A. Krall, and J. B. McBride, Phys. Fluids 28, 538 (1985); S. Riyopoulos, T. Tajima, T. Hatori, and D. Pfirsch, Nucl. Fusion 26, 627 (1986).

${ }^{9}$ R. Hatakeyama, N. Y. Sato, and N. Sato, J. Phys. Soc. Jpn. 60, 2815 (1991); Phys. Rev. E 52, 6664 (1995).
${ }^{10}$ R. A. Demirkhanov, A. G. Kirov, L. F. Ruchko, and A. V. Sukachev, Pis'ma Zh. Eksp. Theor. Fiz. 33, 31 (1981) [JETP Lett. 33, 28 (1981)].

${ }^{11}$ R. Hatakeyama, N. Hershkowitz, and Phaedrus-B Group, in Abstracts of the 44th Annual Meeting of the Physical Society of Japan, Hiratsuka, 1989 (Physical Society of Japan, Tokyo, 1989), No. 4, p. 120.

${ }^{12}$ M. Inutake, M. Ichimura, Y. Kimura, R. Katsumata, H. Hojo, A. Mase, and S. Miyoshi (private communication, 1990).

${ }^{13}$ N. Y. Sato, T. Tanabe, T. Ikehata, H. Mase, R. Hatakeyama, and N. Sato, in Proceedings of the 1994 International Conference on Plasma Physics, Foz do Iguacu, Brazil, 1994, edited by P. H. Sakanaka, E. D. Bosco, and M. V. Alves (INPE, Setor de Eventos, 94ICPP, São José dos Campos, published by American Institute of Physics, New York, 1994), Vol. 3, p. 221.

${ }^{14}$ E. B. Hooper, Jr., R. H. Cohen, D. L. Correll, J. M. Gilmore, and D. P. Grubb, Phys. Fluids 28, 3609 (1985).

${ }^{15}$ J. J. Browning, N. Hershkowitz, T. Intrator, R. Majeski, and S. Meassick, Phys. Fluids B 1, 1692 (1989).

${ }^{16}$ J. J. Browning, R. Majeski, T. Intrator, N. Hershkowitz, and S. Meassick, Phys. Fluids 31, 714 (1988)

${ }^{17}$ K-S. Chung, H. Hutchinson, B. LaBombard, and R. W. Conn, Phys. Fluids B 1, 2229 (1989).

${ }^{18}$ C. S. MacLatchy, C. Boucher, D. A. Poirier, and J. Gunn, Rev. Sci. Instrum. 63, 3923 (1992).

${ }^{19}$ J. R. Ferron, S. N. Golvato, N. Hershkowitz, and R. Goulding, Phys. Fluids 30, 1869 (1987).

${ }^{20}$ D. Segal, Phys. Fluids 18, 640 (1975).

${ }^{21}$ M. Inutake, T. Cho, M. Ichimura, K. Ishii, A. Itakura, I. Katsumata, Y. Kiwamoto, Y. Kusama, A. Mase, S. Miyoshi, Y. Nakashima, T. Saito, A. Sakasai, K. Sawada, I. Wakaida, N. Yamaguchi, and K. Yatsu, Phys. Rev. Lett. 55, 939 (1985).

${ }^{22}$ G. Dimonte, Phys. Rev. Lett. 60, 1390 (1988).

${ }^{23}$ R. A. Breun, N. Hershkowitz, P. Brooker, J. Browning, D. Brouchous, J. R. Conrad, B. J. Cui, D. Edgell, J. Ferron, P. H. Goulding, T. Intrator, D. Keil, R. Majeski, S. Meassick, L. L. Peng, H. Persing, P. Probert, D. Roberts, and Y. Wen, in Plasma Physics and Controlled Nuclear Fusion Research, 1988 (International Atomic Energy Agency, Viena, 1989), Vol. 2, p. 475

${ }^{24}$ See National Technical Information Service Document No. DE 85004960 (B. McVey, "ICRF antenna coupling theory for a cylindrically stratified plasma"). Copies may be ordered from the National Technical Information Service, Springfield, VA, 22161.

${ }^{25}$ A. Tsushima, T. Mieno, M. Oertl, R. Hatakeyama, and N. Sato, Phys. Rev. Lett. 56, 1815 (1986); A. Tsushima and N. Sato, J. Phys. Soc. Jpn. 60, 2665 (1991)

${ }^{26}$ W. Horton, Nucl. Fusion 20, 321 (1980).

${ }^{27}$ T. Hatori and H. Hojo (private communication, 1994). 